\title{
Clinical characteristics and rehabilitation of hospitalised cancer patients in a Korean tertiary hospital
}

\author{
Kyeong Eun $\underline{U h m}^{1}$, MD, Tae Hee $\underline{\text { Yoon }}^{2}$, MD, Ji Hye $\underline{H w a n g}^{2}$, MD, PhD
}

INTRODUCTION With the increase in the patient survival rates of many types of cancers, a greater proportion of cancer patients live with disease-related problems that diminish their quality of life. This study aimed to investigate the clinical characteristics and rehabilitation of hospitalised cancer patients who were referred to the Department of Physical and Rehabilitation Medicine (PRM) at Samsung Medical Center, a tertiary university hospital in Seoul, Korea.

METHODS Hospitalised cancer patients aged $>18$ years who were referred to the Department of PRM from January to December 2012 were enrolled in this retrospective study. We reviewed the clinical characteristics of the patients, the principal reasons for their referral and relevant details of their rehabilitative management.

RESULTS A total of 1,340 cases were included. The most common primary cancer was lung cancer $(19.0 \%)$ and $28.6 \%$ of the cases had solid organ metastasis. The most common reason for referral was deconditioning ( $31.7 \%)$, followed by weakness $(23.1 \%)$ and respiratory problems (14.5\%). Bedside exercise was prescribed to $28.4 \%$ of the patients, exercise in the rehabilitation therapy unit to $28.0 \%$ and pulmonary rehabilitation to $14.3 \%$. Among the 1,340 cases, $107(8.0 \%)$ were transferred to the Department of PRM for comprehensive rehabilitation. The 32 patients with an identifiable Modified Barthel Index score showed significant functional improvement.

CONCLUSION The findings of the present study contribute to a better understanding of rehabilitation for hospitalised cancer patients. The information obtained will also be helpful in the development of appropriate cancer rehabilitation strategies.

Keywords: cancer, inpatients, referral and consultation, rehabilitation

\section{INTRODUCTION}

Cancer has long been a major public health concern. The development of diagnostic tools with increased accuracy and new treatment strategies has increased cancer survival rates. In Korea, more than 200,000 patients are diagnosed with cancer each year and the rate of cancer prevalence per 100,000 exceeds 2,000, according to the 2012 annual report by Korea's Central Cancer Registry and National Cancer Control Institute.(1) The five-year survival rate for all types of cancer in Korea from 2008 to 2012 was $68.1 \% .^{(1)}$

Many patients who are on cancer therapy or have had their cancer treatment terminated have various cancer-related problems that can reduce their quality of life and cause functional deficits. Jo et $\mathrm{al}_{,}{ }^{(2)}$ who used a questionnaire to analyse the physical and psychological problems of 507 cancer patients, reported that $87.1 \%$ of them had physical problems that included pain, numbness, limited range of motion of the joints, weakness, fatigue, lymphoedema, and cognitive, swallowing, speech, language and respiratory problems. Additionally, $48.2 \%$ had one or more psychological problems, including depressive mood and low self-esteem. ${ }^{(2)}$ Another retrospective study involving 159 cancer patients identified the following common impairments (listed in decreasing order of frequency): deconditioning; hemiparesis; cognitive impairment; neurogenic bladder/bowel; cranial nerve deficit; paraparesis; tetraparesis; ataxia; and dysphagia. ${ }^{(3)}$

The significant or permanent functional loss caused by cancer-related problems has been a growing concern since the
$1970 s,{ }^{(4,5)}$ and the rehabilitation needs of cancer patients have been recognised by physicians. ${ }^{(6)}$ In fact, cancer rehabilitation has become one of the subspecialties of rehabilitation medicine. It is concerned with restoring and maintaining the highest possible level of function, independence and quality of life among cancer patients and survivors. ${ }^{(7)}$ Marciniak et al reported that cancer patients who underwent inpatient rehabilitation had significant functional gains (measured using the motor items of the Functional Independence Measure) between admission and discharge. ${ }^{(3)}$ In a prospective study that evaluated the effect of consultation-based interdisciplinary rehabilitation in 290 hospitalised cancer patients, Sabers et al found that more than half of the patients showed functional improvement between enrolment and discharge (58\% as measured using the Karnofsky Performance Status scale and $63 \%$ as measured using the Barthel Mobility Index). ${ }^{(8)}$

The increasing number of cancer patients has led to a greater emphasis on cancer rehabilitation, with the functional status and rehabilitation of cancer patients becoming major issues in cancer treatment. As such, large-scale studies that evaluate the characteristics of hospitalised cancer patients are needed. The present study is a retrospective analysis of the clinical characteristics and rehabilitation of hospitalised cancer patients who were referred to the Department of Physical and Rehabilitation Medicine (PRM) in a Korean tertiary hospital, Samsung Medical Center, Seoul, in the course of one year.

\footnotetext{
'Department of Rehabilitation Medicine, Konkuk University School of Medicine, ${ }^{2}$ Department of Physical and Rehabilitation Medicine, Samsung Medical Center, Sungkyunkwan University School of Medicine, Seoul, South Korea

Correspondence: Dr Ji Hye Hwang, Professor, Department of Physical and Rehabilitation Medicine, Samsung Medical Center, Sungkyunkwan University School of Medicine, 81, Ilwon-ro, Gangnam-gu, Seoul 06351, South Korea. hwanglee@skku.edu
} 


\section{METHODS}

This retrospective chart review involved 1,340 cases of hospitalised cancer patients who were referred to the Department of PRM from January to December 2012. The inclusion criteria were: aged $>18$ years and a diagnosis of cancer, including solid and haematologic malignancy. Patients who were declared cancer-free and hospitalised for reasons not relevant to cancer (e.g. minor surgery) during the study period were excluded from the study. When the same patient was referred more than once during the study period, the consultations were summarised according to the main reasons for each referral and analysed as separate consultation data. When a patient was referred more than once for the same reason, the relevant consultations were analysed as single consultation data.

Patient characteristics including age, gender and the department(s) that requested the patient's consultation with the Department of PRM were collected. Information on the patient's cancer (i.e. type of primary cancer, tumour, node and metastasis [TNM] stage, presence of solid organ metastasis, sites of metastasis, and the time interval between the cancer diagnosis and time of referral) was also examined. The following categories were used for the sites of metastasis: bone, lung, liver, brain, spinal column, others and multiple. The main reason for hospitalisation was also collected and the reasons were categorised as follows: chemotherapy, surgery for primary cancer, management for cancer complications, supportive care and others. The principal reason for referral to the Department of PRM, the management provided and the duration of management were also examined. Principal reasons for referral included deconditioning, weakness, respiratory problem, oedema, pain, dysphagia, pathologic fracture/amputation, joint contracture, bladder/bowel problem, speech/language problem and others. Deconditioning was defined as general weakness caused by prolonged inactivity without a focal neurologic deficit. Weakness was defined as that which was caused by the presence of a central and/or peripheral nervous system lesion, regardless of whether the lesion was primary or secondary to the cancer; it included weakness caused by the presence of a brain or spinal cord tumour, and peripheral nerve entrapment or invasion. Provided management included bedside exercise, exercise in the rehabilitation therapy unit, occupational therapy, physical modality, pulmonary rehabilitation, videofluoroscopic swallowing study (VFSS)/dysphagia therapy, speech/language therapy, complex decongestive physiotherapy (CDPT), nerve conduction study/electromyography (NCS/EMG), fabrication of orthosis/prosthesis, recommendation of medication, education or observation, and others. We also investigated whether the patient had been transferred to the Department of PRM. Modified Barthel Index (MBI) score was used to assess the functional status of the patients who were transferred to the Department of PRM. Wilcoxon signed-rank test was used to determine whether there was any significant difference between the MBI scores of patients before and after rehabilitation. All statistical analyses were conducted using IBM SPSS Statistics for Windows version 20.0 (IBM Corp, Armonk, NY, USA). The study protocol was approved by the International Review Board of Samsung Medical Center (IRB No. 2014-07-106-001).

\section{RESULTS}

A total of 1,340 cases were included in the present study, $786(58.7 \%)$ male and 554 (41.3\%) female. Their mean age was $59.5 \pm 14.0$ years. Most of the referrals to the Department of PRM were made by the Haematology-Oncology (33.3\%) department, followed by the Thoracic and Cardiovascular Surgery (18.3\%), Neurosurgery (10.0\%), General Surgery (9.7\%) and Obstetrics and Gynaecology (8.7\%) departments. Fig. 1 shows the distribution of the referrals across departments.

The primary cancers of the patients who were referred to the Department of PRM are shown in Fig. 2. The most common primary cancers were lung cancer $(19.0 \%)$ and haematologic malignancy (18.0\%). Among the 1,340 cases, the TNM stage was identifiable for 913 (68.1\%) cases; among these cases, $16.3 \%$ were Stage I cancers, $21.7 \%$ were Stage II cancers, $27.9 \%$ were Stage III cancers and $34.1 \%$ were Stage IV cancers. Solid organ metastasis was identified in $383(28.6 \%)$ cases, and the most common site of metastasis was the bone (35.8\% of all metastatic cases), followed by the lungs (31.1\%), brain (29.8\%), liver (19.3\%), spinal column (13.1\%) and others (3.1\%). 95 (24.8\%) of the cases of solid organ metastasis involved multiple solid organ metastases.

The mean time interval between the cancer diagnosis and time of referral to the Department of PRM was $20.0 \pm 31.6$ months. The main reasons for hospitalisation were chemotherapy $(38.4 \%)$, surgery for primary cancer $(30.4 \%)$, management for complications of cancer $(10.1 \%)$, supportive care $(1.5 \%)$ and others $(19.6 \%)$. The most common principal reason for referral was deconditioning $(31.7 \%)$, while other reasons included weakness $(23.1 \%)$ and respiratory problems (14.5\%) (Fig. 3).

The patients were most commonly managed with bedside exercise $(28.4 \%)$ and exercise in the rehabilitation therapy unit $(28.0 \%)$ and pulmonary rehabilitation (14.3\%) (Fig. 4). The mean duration of management was $11.1 \pm 29.5$ days. More than half of the cases (i.e. $51.3 \%$ ) received $\leq 3$ days of management, while $13.1 \%, 15.2 \%$ and $20.3 \%$ received $4-7,8-14$, and $\geq 15$ days of management, respectively.

Among the 1,340 cases who were referred to the Department of PRM, only 107 (8.0\%) were transferred to the department for comprehensive rehabilitation. About one-third of these 107 patients had brain tumour, which was the most common primary cancer among the patients who were transferred to the Department of PRM. Fig. 5 shows the percentage distribution of the primary cancer of these patients. All of the 107 transferred patients received comprehensive rehabilitation for three weeks, unless they had a medical emergency during that period. The MBI score was identifiable in only $32(29.9 \%)$ patients, in whom the mean $\mathrm{MBI}$ score was $26.2 \pm 30.2$ at admission to the Department of PRM and $45.7 \pm 31.4$ at discharge. The difference in the scores before and after rehabilitation was significant $(p<0.001)$, showing that comprehensive rehabilitation at the Department of PRM resulted in significant functional improvement. 


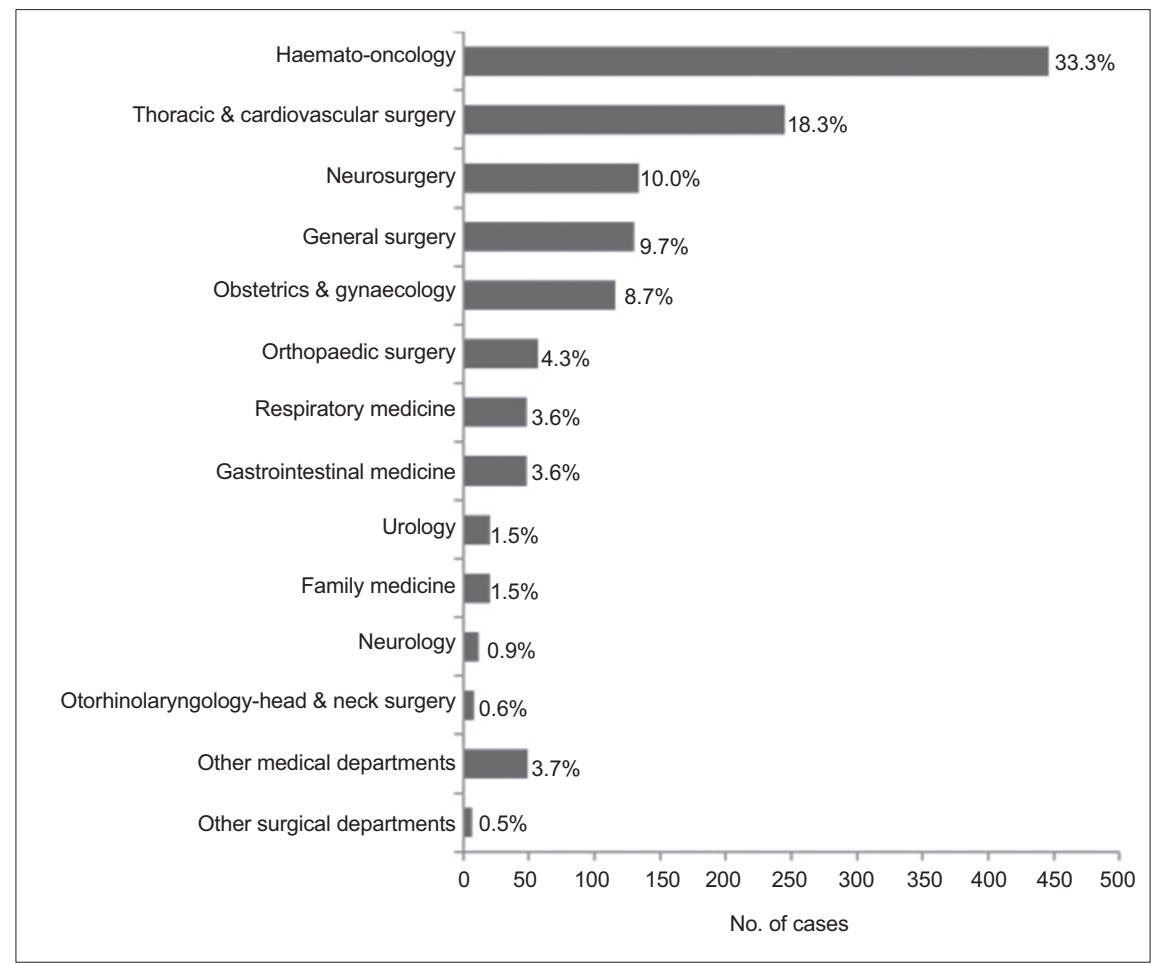

Fig. 1 Bar graph shows the percentage distribution of the departments that made the referrals.

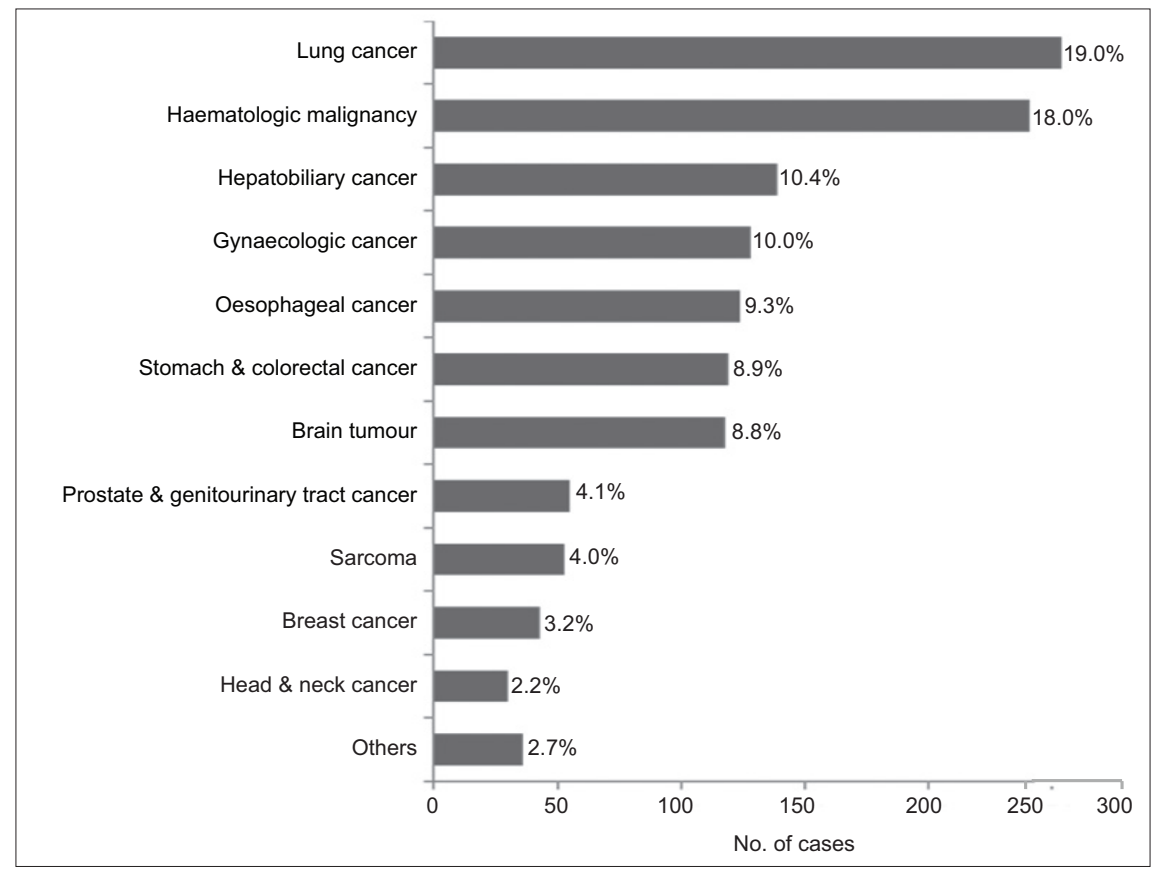

Fig. 2 Bar graph shows the percentage distribution of the primary cancer of the patients who were referred.

\section{DISCUSSION}

At the time of writing, this was the largest study to analyse the characteristics of hospitalised cancer patients who attended consultation-based rehabilitation. The results of our study can help to shed light on the demographic characteristics, functional deficits and other problems that interfere with the quality of life of hospitalised cancer patients.

In our study, the most common primary cancer among the referred cases was lung cancer $(19.0 \%)$, followed by haematologic malignancy $(18.0 \%)$ and hepatobiliary cancer $(10.4 \%)$. In a study by Shin et al, ${ }^{(9)}$ which analysed 1,098 inpatient consultations, the authors reported that haematologic tumour $(24 \%)$ was the most common, followed by primary neurologic tumour $(17 \%)$ and gastrointestinal tumour $(9 \%)$. On the other hand, Sabers et a ${ }^{(8)}$ studied 189 consultation-based rehabilitation cases of hospitalised cancer patients with functional impairment and found that $30 \%$ of the patients had leukaemia or lymphoma, $11 \%$ had lung cancer and $9 \%$ had colon or rectal cancer. The discrepancies observed between the studies may be due to differences in patient inclusion criteria, differences in the distribution of cancer type in the study 


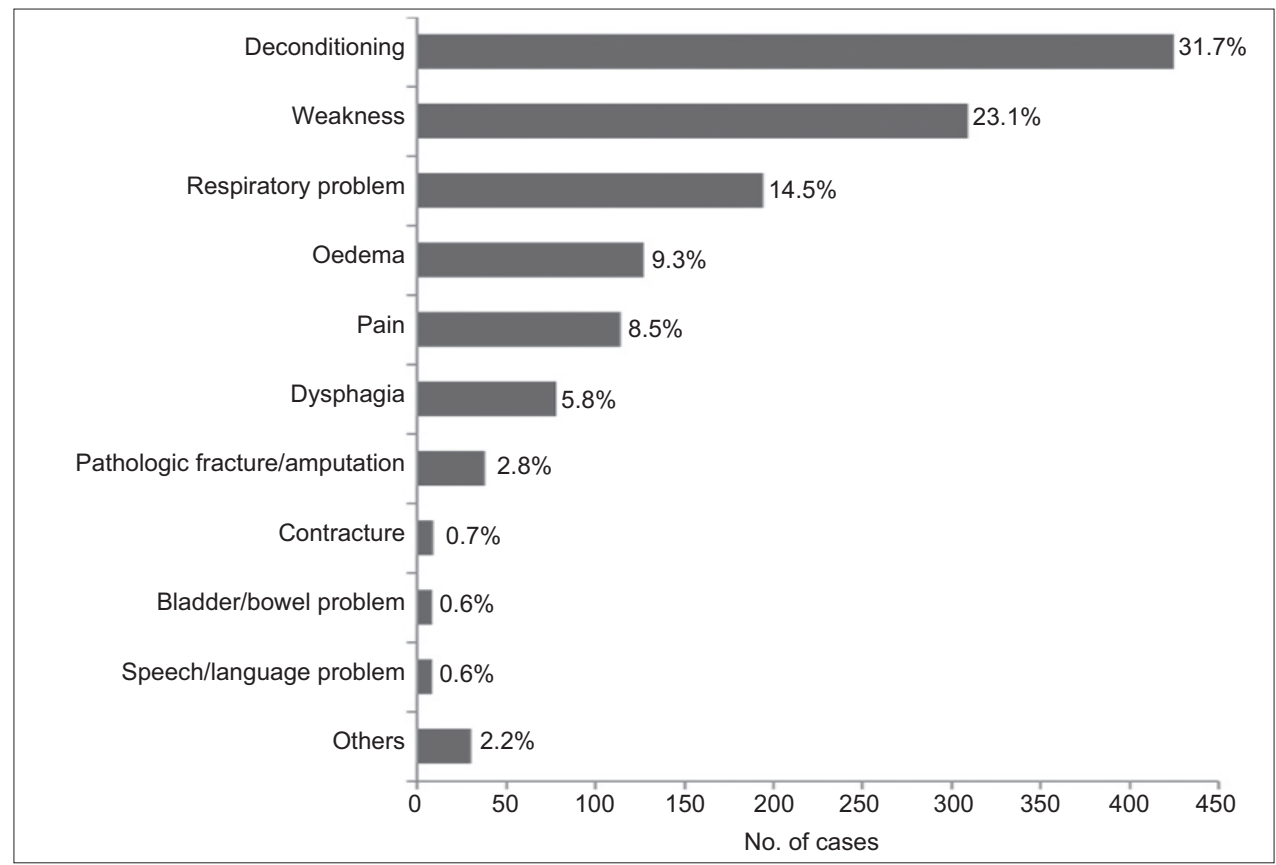

Fig. 3 Bar graph shows the principal reasons for referral.

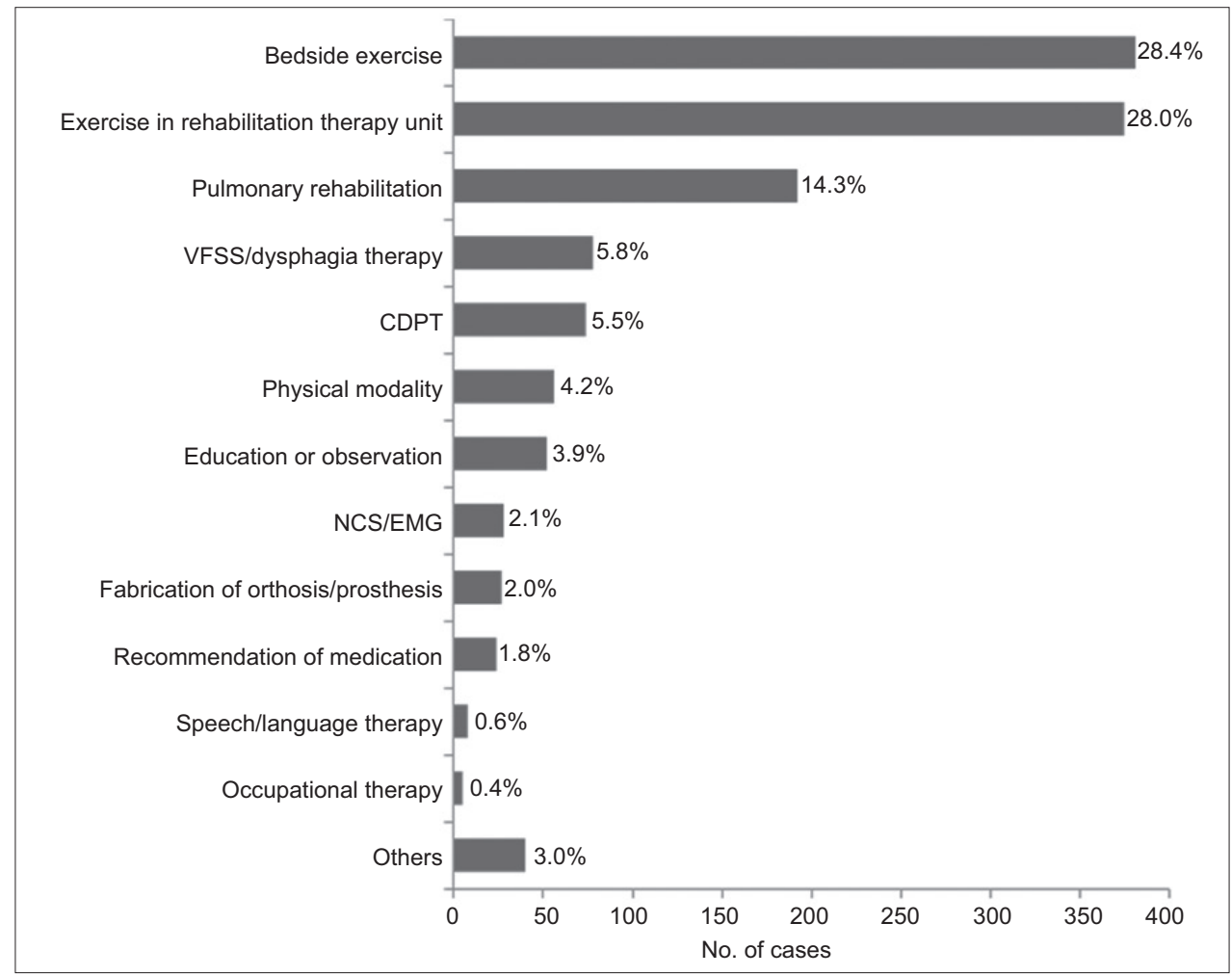

Fig. 4 Bar graph shows the type of management provided to the patients who were referred. CDPT: complex decongestive physiotherapy; NCS/EMG: nerve conduction study/electromyography; VFSS: videofluoroscopic swallowing study

institution and the presence of a rehabilitation oncology unit in some institutions. The present study may also have included many cases of lung cancer (19.0\%) and oesophageal cancer $(9.3 \%)$ due to our hospital's routine practice of enrolling patients who have undergone thoracic surgery into a pulmonary rehabilitation programme. Among the 383 cases of solid organ metastasis, the distribution of the site of metastasis was similar to that reported in a previous study. ${ }^{(8)}$
Cancer patients are at risk for developing various complications resulting from chemotherapy drug toxicity, radiation toxicity, primary and/or secondary nervous system involvement and prolonged immobilisation. In the present study, the most common principal reason for referral to the Department of PRM was deconditioning $(31.7 \%)$, followed by weakness (23.1\%), respiratory problems $(14.5 \%)$ and oedema $(9.3 \%)$. In a study by Marciniak et al involving 159 cancer patients who 


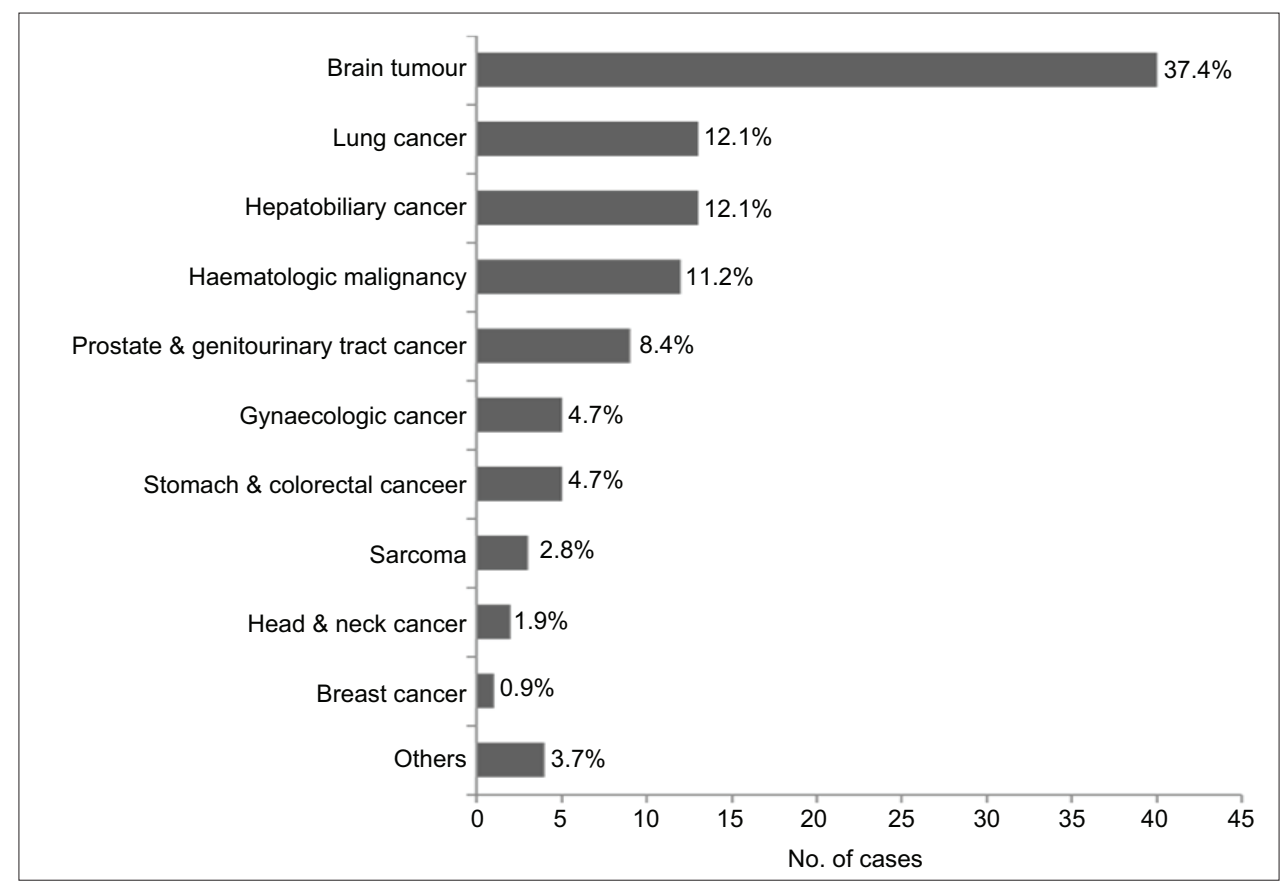

Fig. 5 Bar graph shows the percentage distribution of the primary cancer of the patients who were transferred $(n=107)$.

received inpatient rehabilitation, the most common impairment was deconditioning (30\%), followed by hemiparesis $(29 \%)$, cognitive deficit $(28 \%)$, and neurogenic bladder and bowel $(19 \%) .{ }^{(3)}$ Marciniak et al may have observed a high frequency of neurologic impairments because brain tumour patients took up a large proportion of that cohort. In a prospective study that assessed the rehabilitation needs of 55 cancer patients in an inpatient oncology unit, Movsas et al found that $76 \%$ of the patients showed deconditioning, 58\% showed mobility impairment, $42 \%$ showed a decrease in range of motion and $22 \%$ showed deficits in activities of daily living. ${ }^{(6)}$ Collectively, all these studies, including ours, showed that deconditioning was the most common functional problem among cancer patients who were referred for rehabilitation.

Among the cases that were referred to the Department of PRM, 8.5\% were for pain management. The prevalence of pain among cancer patients increases as cancer progresses. Sabers et $\mathrm{al}^{(8)}$ reported that pain was identified as a significant problem in $74 \%$ of their 189 cancer patients, and Donnelly and Walsh ${ }^{(10)}$ reported that $82 \%$ of their 1,000 advanced cancer patients had pain. In other words, pain is a major, significant problem that needs to be addressed, even in patients whose cancer is not terminal. Primarily, medications are used to control pain. Exercise has also been shown to reduce chronic pain in cancer survivors ${ }^{(11)}$ and could have a positive role in cancer rehabilitation.

In the present study, 107 (8.0\%) patients were transferred to the Department of PRM for comprehensive rehabilitation. Brain tumour, which can induce significant neurological deficits, was the most common type of cancer among these patients $(37.4 \%)$; this is consistent with the findings of previous studies. ${ }^{(3,9)}$

Most of the patients in the present study received bedside exercise $(28.4 \%)$ and exercise in the rehabilitation therapy unit $(28.0 \%)$. Other types of management included pulmonary rehabilitation, dysphagia therapy, CDPT and physical modality. Similar results were found in a retrospective study by Bang et al involving 119 hospitalised patients with cancer. ${ }^{(12)}$ The following rehabilitative procedures were provided for the cases: strengthening exercise (35.3\%), range of motion exercises $(33.6 \%)$, orthosis application $(28.6 \%)$, physical modality $(24.4 \%)$, gait training $(12.6 \%)$, mat exercises $(12.6 \%)$, VFSS and dysphagia training $(9.2 \%)$, observation $(8.4 \%)$ and occupational therapy $(4.2 \%){ }^{(12)}$ The two studies reflect that deconditioning and weakness are the most common problems in this group of patients and that various other problems can cause functional deficits in cancer survivors. Nonetheless, one difference was that pulmonary rehabilitation was more frequently performed in our study, likely because our hospital has a well-established pulmonary rehabilitation programme.

In general, more than half of the cases $(51.3 \%)$ in our study received management for a duration of $\leq 3$ days, reflecting a short length of hospital stay. This means that our study population consisted of patients who were receiving ongoing cancer therapy (e.g. surgery, chemotherapy or radiotherapy) as well as chronic patients. Apart from the aforementioned types of management, other studies have mentioned that procedures such as joint injections and botulinum toxin injections for symptom control ${ }^{(9)}$ and psychosocial support ${ }^{(13)}$ are potential areas of cancer rehabilitation. There is also an increasing emphasis on patient education.

This study has several limitations. First, the degree and severity of functional deficits were not assessed for most of the patients who were transferred to the Department of PRM. Second, the effectiveness of the intervention (i.e. whether the patients who underwent the intervention had functional improvement or not) was not assessed for most of the patients. MBI scores were only 
available for 32 of the 107 patients who were transferred to the Department of PRM for comprehensive rehabilitation. Third, as this was a single-centre study that did not include outpatient data, our results may not be an accurate reflection of the demographic characteristics, symptoms and functional deficits of all cancer patients. Fourth, the results mostly consisted of descriptive data, due to the retrospective design of the study.

To conclude, compared to a decade ago, the annual incidence of all cancers has approximately doubled in Korea; there has also been a change in the distribution of cancer types. ${ }^{(1)}$ With these changes and the establishment of cancer centres in several major tertiary hospitals, the rehabilitation needs of cancer patients have increased. The results of the present study can contribute to a better understanding of cancer rehabilitation for hospitalised cancer patients and may be helpful in the development of appropriate strategies for cancer rehabilitation. The distribution of cancer types and major problems encountered may be different between cancer patients attending outpatient clinics and those who are hospitalised. For example, genitourinary cancer and breast cancer patients account for a large portion of the patients who attend outpatient rehabilitation clinics, and these patients mainly complain of incontinence and lymphoedema. ${ }^{(14)}$ Thus, a different approach is needed when evaluating the functional deficits of cancer patients who attend outpatient clinics and making rehabilitation plans for this group of patients.

\section{REFERENCES}

1. Jung KW, Won YJ, Kong HJ, et al. Cancer statistics in Korea: incidence, mortality, survival, and prevalence in 2012. Cancer Res Treat 2015; 47:127-41.

2. Jo JM, Hwang JH, Lee $\mathrm{CH}$, Kang HJ, Yu JN. The need of cancer patients for rehabilitation services. J Korean Acad Rehabil Med 2010; 34:691-700.

3. Marciniak CM, Sliwa JA, Spill G, Heinemann AW, Semik PE. Functional outcome following rehabilitation of the cancer patient. Arch Phys Med Rehabil 1996; 77:54-7.

4. Mayer NH. Concepts in cancer rehabilitation. Semin Oncol 1975; 2:393-8.

5. Lehmann JF, DeLisa JA, Warren CG, et al. Cancer rehabilitation: assessment of need, development, and evaluation of a model of care. Arch Phys Med Rehabil 1978; 59:410-9.

6. Movsas SB, Chang VT, Tunkel RS, et al. Rehabilitation needs of an inpatient medical oncology unit. Arch Phys Med Rehabil 2003; 84:1642-6.

7. Stubblefield MD. Cancer rehabilitation. Semin Oncol 2011; 38:386-93.

8. Sabers SR, Kokal JE, Girardi JC, et al. Evaluation of consultation-based rehabilitation for hospitalized cancer patients with functional impairment. Mayo Clin Proc 1999; 74:855-61.

9. Shin KY, Guo Y, Konzen B, et al. Inpatient cancer rehabilitation: the experience of a national comprehensive cancer center. Am J Phys Med Rehabil 2011; 90(5 Suppl 1):S63-8.

10. Donnelly S, Walsh D. The symptoms of advanced cancer. Semin Oncol 1995; 22(2 Suppl 3):67-72.

11. Wong P, Muanza T, Hijal T, et al. Effect of exercise in reducing breast and chest-wall pain in patients with breast cancer: a pilot study. Curr Oncol 2012; 19:e129-35.

12. Bang MS, Shin $\mathrm{HI}$, Yoon KJ. Consultation-base rehabilitation management for the hospitalized cancer patients. J Korean Acad Rehabil Med 2003; 27:260-4. Korean.

13. Silver JK, Baima J. Cancer prehabilitation: an opportunity to decrease treatmentrelated morbidity, increase cancer treatment options, and improve physical and psychological health outcomes. Am J Phys Med Rehabil 2013; 92:715-27.

14. Alappattu MJ, Coronado RA, Lee D, Bour B, George SZ. Clinical characteristics of patients with cancer referred for outpatient physical therapy. Phys Ther 2015; 95:526-38. 